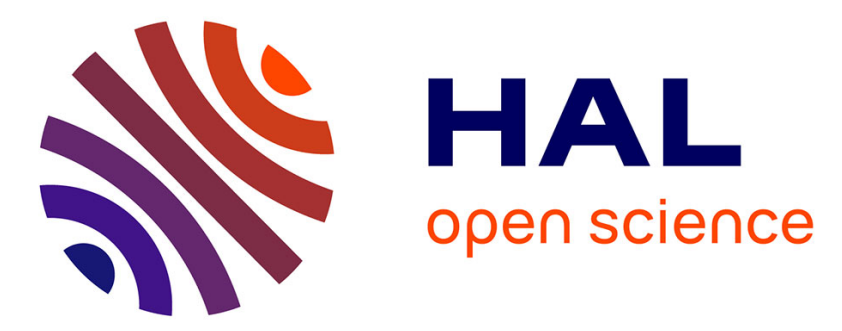

\title{
CALCUL DES EFFETS ANÉLASTIQUES DUS A L'INTERACTION ENTRE DISLOCATIONS ET DÉFAUTS PONCTUELS
}

\author{
P. Peguin, Gilbert Fantozzi, J. Delorme, P. Gobin
}

\section{- To cite this version:}

P. Peguin, Gilbert Fantozzi, J. Delorme, P. Gobin. CALCUL DES EFFETS ANÉLASTIQUES DUS A L'INTERACTION ENTRE DISLOCATIONS ET DÉFAUTS PONCTUELS. Journal de Physique Colloques, 1971, 32 (C2), pp.C2-119-C2-126. 10.1051/jphyscol:1971226 . jpa-00214552

HAL Id: jpa-00214552

https://hal.science/jpa-00214552

Submitted on 1 Jan 1971

HAL is a multi-disciplinary open access archive for the deposit and dissemination of scientific research documents, whether they are published or not. The documents may come from teaching and research institutions in France or abroad, or from public or private research centers.
L'archive ouverte pluridisciplinaire HAL, est destinée au dépôt et à la diffusion de documents scientifiques de niveau recherche, publiés ou non, émanant des établissements d'enseignement et de recherche français ou étrangers, des laboratoires publics ou privés. 


\title{
CALCUL DES EFFETS ANÉLASTIQUES DUS A L'INTERACTION ENTRE DISLOCATIONS ET DÉFAUTS PONCTUELS
}

\author{
P. PEGUIN \\ Centre scientifique et technique du bâtiment \\ G. FANTOZZI, J. F. DELORME et P. GOBIN \\ Département de Physique, Institut National des Sciences Appliquées, \\ 69-Villeurbanne, France
}

\begin{abstract}
Résumé. - Une méthode de calcul numérique est proposée pour décrire le lien entre le cycle effort-déformation, le frottement intérieur et l'anomalie de période (ou de fréquence) d'un matériau dont le comportement est anélastique. Le calcul est développé dans le cas d'un modèle de désancrage thermomécaniquement activé des lignes de dislocations, de leurs ancrages mous, (Peguin-Birnbaum, 1968). Dans ce cas la notion classique d'anomalie de module issue de l'hypothèse de l'élasticité linéaire ne peut être convenablement utilisée et doit être remplacée par la notion d'anomalie de fréquence (ou de période).
\end{abstract}

Abstract. - A numerical calculation is described which allows to relate the stress-strain cycle, the internal friction and the frequency (period) change of a material the behavior of which is anelastic. The calculation was developped in the case of the thermomecanical unpinning of dislocations lines pinned at equidistant point (Peguin-Birnbaum, 1968). The usual notion of modulus change is shown to cannot be used and to be remplaced by the notion of frequency (or period) change.

I. Introduction. - Les solides cristallins réels contiennent des défauts de réseau (défauts ponctuels et linéaires) qui leur confèrent des propriétés anélastiques et plastiques. Ces défauts peuvent avoir deux effets sur les modules élastiques : un effet dû à leur simple présence et un effet dû à leur déplacement.

En effet, la présence de défauts perturbe les liaisons interatomiques et modifie donc les constantes élastiques. Dienes (1952) et Melngailis (1966) ont étudié l'effet de la présence de défauts ponctuels sur le module d'Young. La concentration après déformation, trempe ou irradiation étant en général faible $\left(<10^{-4}\right)$, l'effet de volume est ainsi négligeable. Il en est de même pour la présence des dislocations. Par contre, les effets dus aux mouvements de défauts sont beaucoup plus importants. Ces effets sont également extrêmement complexes car ils font appel non seulement aux caractéristiques propres du défaut envisagé, mais aussi à des interactions à courte et grande distance avec les autres défauts du réseau.

Sous l'influence d'une contrainte appliquée que l'on supposera par la suite sinusoïdale, les défauts peuvent contribuer à la déformation de manière réversible ou irréversible, après suppression de la contrainte, apparaît alors une déformation rémanente $\varepsilon_{p}$ que đans le cadre de ce travail nous supposerons toujours nulle.

La déformation réversible peut être élastique ou anélastique. Il y a élasticité (linéaire ou non linéaire) si la contrainte et la déformation sont reliées de façon biunivoque. Dans le cas contraire, il y a anélasticité
(Zener, 1955). En effet, la non-biunivocité de la fonction d'état liant l'effort $\sigma$ à la déformation $\varepsilon$ se traduit par la présence d'un cycle dans le plan $\sigma \varepsilon$.

Dans l'hypothèse de l'élasticité linéaire, l'éprouvette subit des contraintes et des déformations sinusoïdales de pulsation $\omega$ déterminée par la résolution de l'équation différentielle linéaire à coefficients constants du système appareil de mesure-éprouvette. La fréquence $f$ est alors proportionnelle à la racine carrée du module du matériau $E$ :

$$
E=k f^{2}
$$

Si la déformation macroscopique d'un solide réel contenant des défauts conserve un comportement élastique linéaire, on peut définir une variation de module :

$$
\begin{aligned}
\frac{\Delta E}{E_{e}}=\frac{E-E_{e}}{E_{e}}=\frac{-\varepsilon_{d}}{\varepsilon_{d}+\varepsilon_{e}}=\frac{2\left(f-f_{e}\right)}{f_{e}} & = \\
= & -2 \frac{T-T_{e}}{T_{e}}
\end{aligned}
$$

$\varepsilon_{e}:$ déformation élastique pure.

$\varepsilon_{d}$ : déformation réversible due aux mouvements des défauts.

$E_{e}$ : module du matériau sans défaut.

$\Delta E / E_{e}=$ anomalie de module.

$T=1 / f$ période du mouvement.

$E$ : module dú matériau avec défauts. 
Cette formule peut être appliquée, par exemple, en première approximation au cas des dislocations tant qu'il n'y a pas désancrage et pour des fréquences assez basses pour que l'amortissement visqueux dû aux interactions dislocations-phonon soit négligeable. La déformation $\varepsilon_{d}$ due aux dislocations est alors donnée par une formule de la forme (Mott, 1952, Friedel, 1953, Granato et Lucke, 1956)

$$
\varepsilon_{d}=\frac{\sigma l^{3}}{6 G}
$$

\section{$l$ : longueur des boucles.}

Si le matériau possède un comportement élastique non linéaire, le module instantané varie avec l'état de contrainte. On peut montrer dans ce cas que la définition précédente de l'anomalie de module est toujours valable à condition toutefois que les variations du module ne soient pas trop importantes (inférieures à $15 \%$ ) et que l'on précise la valeur maximale de la contrainte atteinte au cours du mouvement (Delorme et al., 1970).

La formule (1) a été utilisée par de nombreux auteurs. En fait, elle ne constitue qu' une première approximation de la réalité physique car elle n'est applicable qu'à l'élasticité. Dans le cas des effets anélastiques réversibles et des effets irréversibles, les notions d'anomalie de période et d'anomalie de module doivent être clairement distinguées. On peut se demander quelle est la signification physique du module au cours d'un cycle de forme quelconque. Seule nous est accessible expérimentalement la période du cycle qui reflète les lois de variation de la contrainte et de la déformation en fonction du temps. Ces lois ne sont plus parfaitement sinusoïdales car l'équation différentielle du mouvement n'est plus linéaire à coefficient constant. $\mathrm{La}$ résolution mathématique générale du problème dans le cas d'un modèle physique donné est extrêmement complexe, voire même impossible. On doit faire alors appel au calcul numérique.

On peut cependant relier simplement anomalie de période et anomalie de module lorsque les cycles sont réguliers et symétriques par rapport à l'origine (certains cas de plasticité) ou possèdent un axe de symétrie. La pente $E$ de cet axe donnera le module moyen. En visco-élasticité linéaire, le cycle $\sigma-\varepsilon$ elliptique permet de définir un module égal à la pente du grand axe de l'ellipse. Physiquement il représente le rapport de la contrainte à la déformation qui est en phase avec elle. Le module est donné en fonction de la fréquence par une relation du même type que celle qu'on obtient dans le cas de l'élasticité linéaire : $E=k f^{2}$. Dans le cas d'un pic de Debye, on peut donc parler d'effet $\Delta E$.

Si le cycle n'est pas symétrique, la notion de module, et par conséquent d'anomalie de module, perd cette signification simple. Il faut parler de période (ou de fréquence) et d'anomalie de période ou de (fréquence).

Pour interpréter certaines expériences de frottement intérieur, il nous a paru utile de mettre au point une méthode de calcul numérique qui permet d'obte- nir l'anomalie de période, le frottement intérieur et de tracer le cycle effort-déformation pour un modèle physique déterminé. Nous avons choisi le phénomène de désancrage thermomécanique des lignes de dislocations piégées (modèle de Peguin-Birnbaum, 1968). Il nous a permis de calculer l'anomalie de période ou de fréquence associée aux cycles effort-déformation et le frottement intérieur en fonction de la température, de la déformation et de la fréquence d'oscillation. Le calcul a été effectué dans le cas d'oscillations de torsion.

II. Modèle d'anélasticité utilisé. - La méthode de calcul que nous présentons dans la troisième partie est valable pour des modèles physiques ou rhéologiques d'anélasticité réversible pour lesquels on peut définir en chaque point du cycle effort-déformation un «module instantané » $\sigma / \varepsilon$, correspondant à l'état du matériau en ce point. Nous avons choisi de développer le cas du désancrage thermomécaniquement activé d'une ligne de dislocation de ses ancrages mous.

II.1 LE FROTTEMENT INTÉRIEUR DU TYPE $\Delta H$. La croissance du frottement intérieur avec l'amplitude de la déformation a été observée depuis longtemps (Read, 1940). Granato et Lucke (1956) ont interprété cette augmentation comme un dépiégeage purement mécanique des lignes de dislocations. Un tel modèle qui fait une distinction entre des ancrages «durs » et des ancrages "mous», conduit à deux types de frottement interne:

a) La ligne piégée se comporte comme une corde vibrante et il y a absorption d'énergie par un phénomène de résonance. Il s'agit de $\Delta I$ qui atteint sa valeur maximale dans le domaine de fréquence du mégacycle (Stern, Granato, 1962). Il a été montré que $\Delta I$ est indépendant de l'amplitude de la contrainte. Par ailleurs $\Delta I$ varie comme $\Lambda L^{4}(\Lambda=$ densité de dislocation, $L=$ longueur de la ligne), aussi au moment du. désancrage l'augmentation de longueur de ligne libre s'accompagne d'un changement de niveau de $\Delta I$ vers des valeurs plus élevées (Rogers, 1962, Mason, 1966, Tittman, Bommel, 1966, Hikata, Elbaum, 1967).

b) Le désancrage des lignes de dislocations sous l'action de la contrainte, s'accompagne d'une absorption d'énergie qui passe par un maximum en fonction de la contrainte appliquée, c'est le $\Delta H$ (Granato, Lucke, 1956). La mise en évidence expérimentale de ce frottement intérieur est souvent contrariée par la rapide croissance de l'énergie dissipée aux amplitudes plus élevées (Peguin, Perez, Gobin, 1967). Il est cependant possible dans certains cas d'isoler le maximum de $\Delta H$.

C'est le deuxième type de frottement intérieur, c'està-dire le $\Delta H$ que nous considérons dans ce qui suit, en gardant à l'esprit qu'aux fréquences de mesures élevées $\left(>10^{4} \mathrm{c} . \mathrm{s}^{-1}\right), \Delta I$ et sa variation au moment du désancrage ne peuvent plus être négligées. 
II. 2 ModÈle D'ANÉLASTrCITÉ UTILISÉ. - Nous rappellerons brièvement les bases du modèle numérique mis au point précédemment, et qui utilise les relations développées par Teutonico et al. (1964) et Heuser (1965) pour décrire le désancrage thermomécaniquement activé d'une ligne de dislocation piégée, ces auteurs ont supposé que l'interaction défaut-dislocation pouvait être décrite par une fonction de CottrellBilby linéarisée. Ils ont ainsi pu calculer l'énergie potentielle $\mathrm{du}$ système soumis à une contrainte en fonction de la distance dislocation-ancrage. Leurs calculs montrent qu'en dessous d'une contrainte critique $\sigma_{2}$ ou contrainte de dépiégeage thermique, il n'existe qu'une position d'équilibre pour la ligne: la position piégée. Au-dessus d'une contrainte critique appelée $\sigma_{1}$ ou contrainte de dépiégeage mécanique, il n'existe également qu'une position d'équilibre : la position dépiégée. Pour $\sigma_{2}<\sigma<\sigma_{1}$, la ligne peut sauter, sous l'action d'une activation thermomécanique, d'une position d'équilibre stable piégée à une position d'équilibre stable dépiégée ou inversement. Mais cette analyse ne permet pas facilement d'aboutir au calcul du frottement intérieur dû au comportement global de la ligne piégée en plusieurs points. Par un modèle numérique de calcul (Peguin et Birnbaum, 1968), il a été possible d'étendre cette étude au calcul complet des cycles effort-déformation et du frottement intérieur dû au désancrage thermomécanique des lignes de dislocations. Ce calcul a pu être réalisé en faisant varier successivement les paramètres suivants : contrainte appliquée, température, fréquence, nombre d'ancrages, énergie d'interaction ligne-ancrage.

Pour cela, on considère une ligne de dislocation ayant $N_{0}$ points d'ancrage équidistants (Fig. 1). En configuration 1 c'est-à-dire à l'état complètement

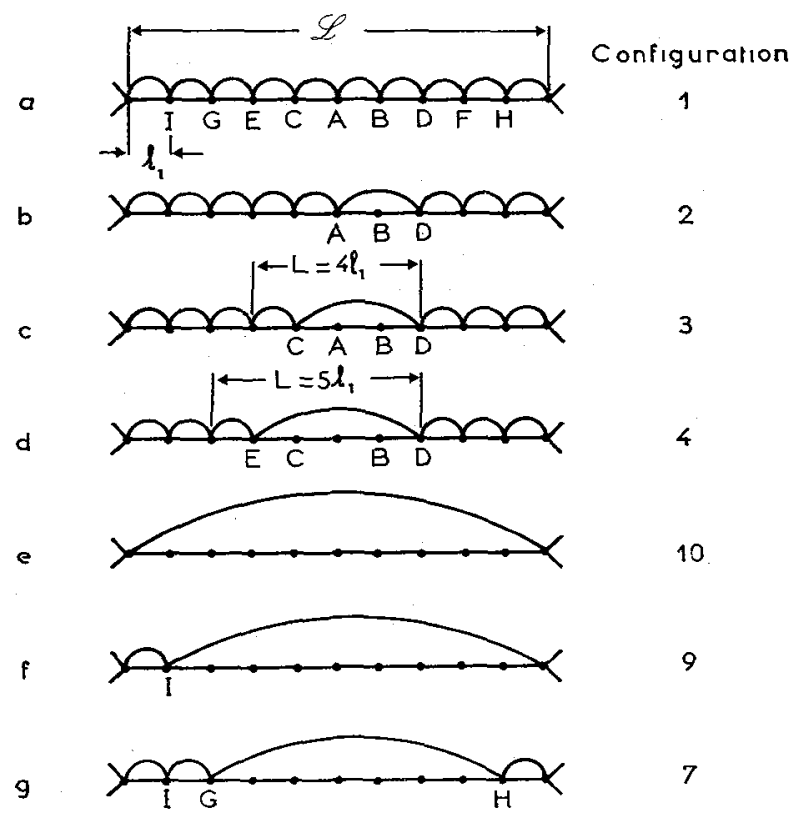

FIG. 1. - Différentes configurations de la ligne de dislocation du modèle utilisé. piégé, on peut étudier le comportement de cette ligne en supposant qu'elle est formée de $N_{0}$ boucles ayant un ancrage en leur milieu. Le dépiégeage et le repiégeage thermomécaniquement activé de chacune de ces boucles est connu (Teutonico et al., 1964). Si l'une de ces boucles est à l'état dépiégé, la ligne se trouve en configuration (2) sur la figure $1 b$. Nous pouvons alors étudier la boucle $\mathrm{CD}$ (ou AF) ayant un ancrage au tiers de sa longueur. Son comportement est également connu, nous pouvons donc connaître la probabilité de dépiégeage de A (ou D) afin d'obtenir la configuration (3) et ainsi de suite jusqu'en la configuration correspondant au dépiégeage complet.

Plus généralement, il a été montré qu'une ligne en configuration $(J)$, c'est-à-dire étant dépiégée de $(J-1)$ points d'ancrage successifs a une fréquence de sauts vers la configuration $(J+1)$ donnée par :

$$
F u(J)=\alpha v u(J) P u(J)
$$

$\alpha$ est un coefficient un peu inférieur à 2 qui tient compte du fait que la boucle peut se dépiéger à ses deux extrémités (sauf pour le saut de la configuration 1 à 2 où $\left.\alpha=N_{0}\right)$.

$v u(J)$ est la fréquence de vibration de la ligne, donnée par la relation habituelle $v D \frac{b}{L(J)}(v D=$ fréquence de Debye, $b=$ vecteur de Burgers, $L(J)=$ longueur libre de la ligne). Ceci en négligeant le terme d'entropie (Vineyard, 1957) qui pourtant a été trouvé important dans un cas simple (Granato, Lucke, Schlipf, Teutonico, 1964).

$P u(J)$ est la probabilité de dépiégeage de $(J)$ en $(J+1)$; ce terme est de la forme $\exp \left(-U_{u}(J) / k T\right)$ où $U_{u}(J)$ est l'énergie nécessaire au passage de $(J)$ à $(J+1)$. De même la fréquence de saut de la configuration $(J)$ à la configuration $(J-1)$ est donnée par

$$
F_{R}(J)=\beta v_{R}(J) P_{R}(J) .
$$

$\beta$ coefficient égal à 2 qui traduit le fait que la boucle peut se repiéger près de ses extrémités.

$v_{R}(J)$ : fréquence de vibration de la ligne.

$P_{R}(J)$ : probabilité de repiégeage de $J$ en $(J-1)$.

$$
P_{R}(J)=\exp -\frac{U_{R}(J)}{k T} .
$$

Les énergies d'activation correspondant respectivement au dépiégeage et au repiégeage de la configuration $(J)$ vers les configurations $(J+1)$ et $(J-1)$ sont de la forme :

$$
\begin{aligned}
& U u(J)=\frac{U_{0} n[\beta(J)+1]}{\beta(J) n-1}\left(1-\frac{\sigma}{\sigma_{1}(J)}\right)^{2} \\
& U r(J)=\frac{U_{0} n q^{2}}{\beta(J) n-1}\left(\frac{\sigma}{\sigma_{2}(J)}-1\right)^{2}
\end{aligned}
$$

où $n, q$ sont des constantes : $\beta(J), \sigma_{1}(J), \sigma_{2}(J)$ peuvent être déterminées pour chaque configuration.

$U_{0}$ : énergie de liaison maximale entre la dislocation et l'ancrage. 
En multipliant $F u(J)$ et $F_{R}(J)$ par le nombre de lignes dans chaque configuration $(J)$, il est alors possible de calculer à la fin d'un intervalle de temps $\mathrm{d} t$, Ia nouvelle répartition des lignes entre les différentes configurations (on suppose que $\mathrm{d} t$ est suffisamment petit pour que les lignes en configuration $(J)$ n'aient pas le temps de sauter à la configuration $(J \pm 2)$. Si $A N(J)$ est le nombre de boucles en configuration $J$ à l'instant $t$, le nombre de boucles en configuration $J$ à l'instant $t+\mathrm{d} t$ est (Peguin, Birnbaum, 1968) :

$X N(J)=A N(J)+\left(X_{1}+X_{2}-X_{3}-X_{4}\right) \mathrm{d} t$

$X_{1}(J)$ : vitesse de désancrage de $(J-1)$ à $J$.

$X_{2}(J)$ : vitesse d'ancrage de $(J+1)$ à $J$.

$X_{3}(J)$ : vitesse de désancrage de $J$ à $(J+1)$.

$X_{4}(J)$ : vitesse d'ancrage de $J$ à $(J-1)$.

On peut montrer par ailleurs que l'accroissement de déformation due aux boucles qui passent de la configuration $(J)$ à $(J+1)$ pendant le temps $\mathrm{d} t$ est :

$$
\begin{aligned}
\mathrm{d} \varepsilon=\frac{8 b^{2} \sigma l_{1}^{3}}{\pi^{4} C_{t}} \cdot 3(J) \cdot(J & +1) \times \\
& \times\left(X_{3}(J)-X_{2}(J)\right) \cdot \mathrm{d} t .
\end{aligned}
$$

$C_{t}=$ tension de ligne.

Ceci n'est valable que pour une contrainte appliquée telle que $\sigma_{2}(1)<\sigma<\sigma_{1}(1)$. En effet, pour $\sigma<\sigma_{2}(1)$, les boucles de dislocation seront toutes dans la configuration 1. Pour $\sigma>\sigma_{1}(1)^{*}$ les boucles seront dans la configuration $\left(N_{0}+1\right)$ : état désancré.

Remarque. - Il est également possible d'utiliser d'autres hypothèses que celles de Teutonico, Granato et Lucke (1964) pour exprimer l'énergie de désancrage et de réancrage. En particulier, nous avons utilisé un désancrage de type Friedel (1963) qui avait montré la nécessité de tenir compte de l'activation thermique. Dans ce cas, les fréquences de saut de la configuration $J$ à la configuration $(J+1)$ sont données par :

$$
\begin{aligned}
& F \mathbf{u}(J)=\alpha v u(J) \exp \left(-\frac{U_{0}-\sigma b^{2}(J+1) \frac{l}{2}}{k T}\right) \\
& F_{R}(J)=0 .
\end{aligned}
$$

Ceci en supposant que le réancrage n'a lieu que pour $\sigma=0$, donc la fréquence de saut de la configuration $J$ à la configuration $(J-1)$ est nulle. Dans ce cas, l'accroissement de déformation due aux boucles qui passent de la configuration $J$ à $(J+1)$ pendant le temps $\mathrm{d} t$ reste décrit par la relation (6), mais en faisant $X_{2}(J)=0$. La seule limitation de contrainte est cette fois-ci, le désancrage mécanique. Pour $\sigma>\sigma_{1}(1)$, les boucles seront toutes dans la configuration $\left(N_{0}+1\right)$.

* - La contrainte $\sigma_{1}$ diminue quand la longueur libre des lignes de dislocation augmente (c'est-à-dire quand $J$ augmente : $L(J)=(J+1) l_{1}$.

Par conséquent, si $\sigma>\sigma_{1}(1)$ il est fortiori $>\sigma_{1}(J)$.
III. Principe du calcul. - Afin de simplifier le calcul, nous supposons dans le premier temps que l'éprouvette est un tube suffisamment mince pour que la contrainte ou la déformation puissent être considérées comme identiques dans tout le volume. Cette simplification a du reste déjà été utilisée expérimentalement (Morrow, 1965). Le mouvement de l'oscillateur constitué par le tube mince et son dispositif de mise en mouvement est décrit par l'équation différentielle :

$$
I \theta^{\prime \prime}+C \theta=0
$$

où $I$ est le moment d'inertie du système,

$C$ est le couple de rappel.

Comme dans la plupart des méthodes de calcul numérique, on divise le paramètre principal qui est ici le temps, en intervalles suffisamment petits pour que certaines approximations soient valables. La validité de ces approximations étant du reste toujours vérifiée par une série de tests.

Considérons le premier échelon de temps $\mathrm{d} t$ à partir de $t=0$. Nous nous plaçons dans l'hypothèse d'un phénomène de désancrage thermomécanique des lignes de dislocations, le matériau étant purement élastique si ce phénomène ne se manifeste pas. Si le module initial de l'échantillon est $G_{1}$ (couple $C_{1}$ ). On peut calculer le mouvement du pendule au cours de l'échelon $\mathrm{d} t_{1}$ de l'équation (5) par l'algorithme suivant (Timoshenko, 1954).

$$
\begin{aligned}
\theta_{1}^{\prime} & =\theta_{0}^{\prime}+\theta_{0}^{\prime \prime} \mathrm{d} t \\
\downarrow & \\
\rightarrow & \theta_{1}=-\theta_{0}+\frac{\theta_{0}^{\prime}+\theta_{1}^{\prime}}{2} \mathrm{~d} t \\
\downarrow & \\
\theta_{1}^{\prime \prime} & =-\frac{C_{1}}{I} \theta_{1} \\
\downarrow & \\
-\theta_{1}^{\prime} & =\theta_{0}^{\prime}+\frac{\theta_{0}^{\prime \prime}+\theta_{1}^{\prime \prime}}{2} \mathrm{~d} t .
\end{aligned}
$$

Pour effectuer ce calcul il faut connaître $\theta_{0}, \theta_{0}^{\prime}, \theta_{0}^{\prime \prime}$. On a choisi l'origine du mouvement à la position d'équilibre de l'oscillateur.

$$
\theta_{0}=\theta_{0}^{\prime \prime}=0 \text {. }
$$

Différentes valeurs ont été données à $\theta_{0}^{\prime}$ selon l'amplitude du mouvement que l'on désire atteindre (contrainte maximum appliquée). D'autre part le rapport $C_{1} / I$ a été choisi à l'origine tel que la fréquence dans l'hypothèse d'un matériau élastique soit de $10^{4} \mathrm{~Hz}$.

$$
\frac{1}{2 \pi} \sqrt{\frac{C_{1}}{I}}=10^{4} \text {. }
$$

La boucle de calcul est interrompue dès que la valeur de $\theta_{1}$ converge avec une tolérance fixée. On peut alors en déduire une contrainte correspondante : 


$$
\sigma_{1}=G_{1} \theta_{1} \frac{D}{2 l}
$$

$D$ : diamètre du tube $D=100 \mathrm{~mm}$.

$l$ : longueur du tube $l=5 \mathrm{~mm}$.

$G_{1}=10^{12}$ dynes par $\mathrm{cm}^{2}$.

Jusqu'à maintenant on a supposé que le matériau avait un comportement élastique linéaire (le couple $C_{1}$ était constant). Supposons que le phénomène de désancrage des lignes de dislocation intervienne. En supposant que la contrainte ne varie pas au cours d'un petit échelon de temps $\mathrm{d} t$ (on prend comme valeur de la contrainte la moyenne entre les valeurs au début et à la fin de l'échelon de temps $\mathrm{d} t$ ) nous avons vu que, dans le cas du modèle de désancrage utilisé, un certain nombre de lignes de dislocation passe de la configuration $J$ à $J+1$ et donnent ainsi un accroissement de déformation $\mathrm{d} \varepsilon$ qui est fonction de la contrainte, de la température, de la fréquence et qui dépend du modèle physique ou rhéologique choisi.

Dans le cadre de ce travail, d $\varepsilon$ est donné par la relation (6). Le diagramme $(\sigma, \varepsilon)$ correspondant est donné par la figure 2 .

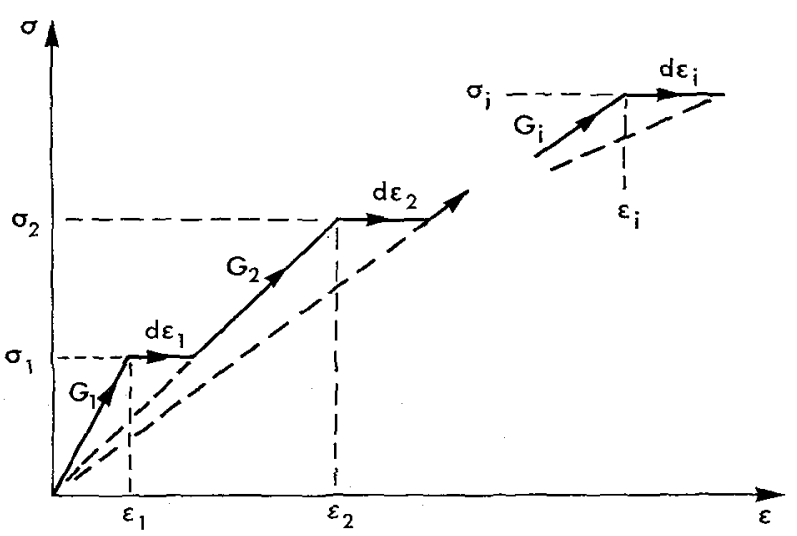

Fig. 2. - Diagramme $(\sigma, \varepsilon)$ au cours des échelons de temps successifs $\left(\mathrm{d} t_{1}, \mathrm{~d} t_{2} \ldots, \mathrm{d} t_{i} \ldots \ldots\right)$.

Pour la valeur de la contrainte $\sigma_{1}$, on aura donc une déformation supplémentaire:

$$
\mathrm{d} \varepsilon_{1}=\frac{24 b^{2} \sigma_{1} l_{1}^{3}}{\pi^{4} C_{t}}\left[\sum_{J=1}^{N_{0}} J(J+1)\left(X_{3}-X_{2}\right)\right] .
$$

Considérons maintenant l'échelon de temps suivant $\mathrm{d} t_{2}$. Le matériau a un comportement élastique linéaire avec un nouveau module $G_{2}$ (compte tenu de la déformation due aux dislocations).

$$
G_{2}=\frac{\sigma_{1}}{\frac{\sigma_{1}}{G_{1}}+\mathrm{d} \varepsilon_{1}}
$$

d'où le couple

$$
C_{2}=C_{1} \cdot \frac{G_{2}}{G_{1}}
$$

Le calcul peut alors se poursuivre. L'algorithme (8) où 1 'on a remplacé les indices 0 et 1 par les indices $i$ et $\mathrm{i}+1$ permet à partir des valeurs de $\theta_{\mathbf{i}}, \theta_{\mathbf{i}}^{\prime}, \theta_{\mathbf{i}}^{\prime \prime}$ et $\mathrm{C}_{\mathbf{i}}$ de calculer $\theta_{\mathbf{i}+1}, \theta_{\mathbf{i}+1}^{\prime}, \theta_{\mathbf{i}+1}^{\prime \prime}, \sigma_{\mathrm{i}+1}$.

On peut alors connaître la déformation supplémentaire $\mathrm{d} \varepsilon_{\mathrm{i}+1}$ due au désancrage et le nouveau module $G_{i+2}$. Le calcul continue jusqu'à ce que $\theta$ repasse par la valeur 0 . On connait alors la demi période du mouvement.

La comparaison du temps mis pour que $\theta$ s'annule avec la valeur de la demi-période du matériau-parfaitement élastique donne l'anomalie de période $\Delta T$. Il est également possible tout au long du calcul de tracer point par point le diagramme effort-déformation, son allure donne des renseignements précieux sur les conséquences du modèle atomique choisi (Roberts, Hartman, 1964) et son aire rapportée à l'énergie élastique mise en jeu donne le frottement interne correspondant.

Cette méthode de calcul, s'applique au modèle Peguin-Birnbaum, présenté au chapitre précédent. Nous avons utilisé le désancrage type Friedel décrit par les relations 7 et nous avons étudié le frottement intérieur, l'anomalie de période et les courbes $\sigma, \varepsilon$ en fonction de la température et de la contrainte pour différentes énergies d'interaction défaut-ligne, longueur de lignes et nombre d'ancrages.

IV. Résultats obtenus. Discussion. - Nous présenterons sur la figure 3 les résultats obtenus pour une

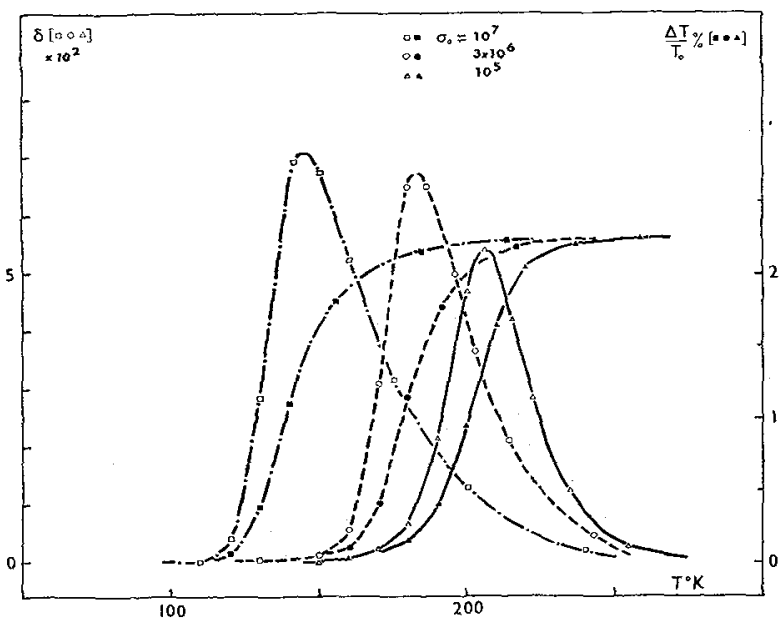

FiG. 3. - Evolution simultanée du frottement intérieur et de l'anomalie de période en fonction de la température dans le cas d'un désancrage de type Friedel (pour différentes contraintes maximales appliquées $\sigma_{0}$ ).

configuration dislocations-défauts ponctuels caractérisée par les données suivantes:

Energie d'interaction défaut ponctuel-dislocation $U_{0}=0,2 \mathrm{eV}$.

Longueur de la ligne de dislocation entre deux ancrages durs $L=1,5 \times 10^{-4} \mathrm{~cm}$. 
Nombre d'ancrages mous entre deux ancrages durs $N_{0}=8$.

Densité d'éléments : $10^{11} \mathrm{~cm}^{-3}$.

Contraintes maximales appliquées $\sigma_{0} 10^{7}, 3 \times 10^{6}$, $10^{5}$. Baryes.

Comme Peguin et Birnbaum (1968) l'avaient observé avec un désancrage de type TGL, le pic de frottement intérieur est asymétrique en température (d'autant plus que $\sigma_{0}$ est grand). De plus, on peut observer qu'au maximum de frottement intérieur, l'anomalie de période est égale à environ $65 \%$ de sa valeur maximale. Le rapport $r=\frac{\delta_{\max }}{\left(\Delta T / T_{0}\right)_{\max }}$ varie avec la contrainte maximale. Il passe de 2,4 à 3,1 quand $\sigma_{0}$ varie de $10^{5}$ baryes à $10^{7}$ baryes. Dans le cas d'un pic de Debye, ce rapport est égal à $\pi$ tandis que dans le modèle de Koiwa-Hasiguti (1965), il est égal à 0,8.

Il faut remarquer que ce rapport $r$ souvent utilisé n'est pas très significatif. En effet il fait intervenir la valeur maximum de $\delta$ et l'écart relatif entre les valeurs de la période avant et après le pic de frottement intérieur. Le rapport $r$, s'il est facile à obtenir, ne peut en aucun cas caractériser un modèle. Il semblerait préférable, pour caractériser la forme des cycles effortdéformation, c'est-à-dire un modèle donné, de tracer la variation continue du rapport $y=\frac{\delta}{\Delta T / T}$ en fonction du paramètre variable (température, contrainte appliquée, fréquence). Le rapport $y$ doit permettre dans ce cas de comparer le modèle étudié au modèle de relaxation pure de Zener dans lequel on sait que :

$$
y=2 \omega T_{0} \exp \frac{\mathrm{Q}}{k \theta} \text {. }
$$

Nous avons effectué cette étude. Sur la figure 4 , on a tracé :

$$
\log \frac{\delta}{\Delta T / T}=f\left(\frac{1}{\theta}\right)
$$

pour les courbes correspondant à la figure 3 . Dans le modèle de Zener on obtient une droite. La figure 4 montre qu'il n'en est pas ainsi dans le modèle que nous avons étudié.

Sur la figure 5 nous présentons des cycles effortdéformation dus aux dislocations pour trois températures différentes, pour une contrainte maximale de $3 \times 10^{6}$ baryes.

L'aire du cycle est maximale à la température $\mathrm{du}$ pic $\left(T \simeq 181^{\circ} \mathrm{K}\right)$. Les cycles sont tous compris entre deux droites extrêmes correspondant aux très basses températures ou très hautes températures (module avec toutes les dislocations désancrées ou module sans dislocations désancrées).

L'étude des 2 figures 3 et 5 permet de vérifier sur ce modèle physique qu'on ne doit pas identifier anomalie de module et anomalie de période. D'après les résultats de la figure 3 on peut connaître l'anomalie de période correspondant à des cycles d'amplitude

$$
\sigma_{0}=3 \times 10^{6} \text { baryes }
$$

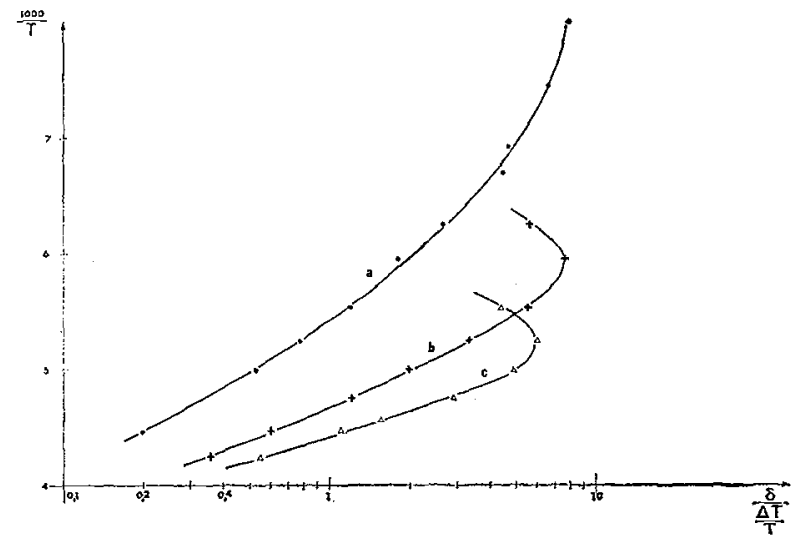

FIG. 4. - Etude de la fonction $\log \frac{\delta}{\Delta T / T}=f(\theta)$ pour le pic correspondant aux trois contraintes.

$$
\begin{aligned}
\text { I }-\sigma_{0} & =10^{7} \text { baryes } \\
\text { II }-\sigma_{0} & =3 \times 10^{6} \text { baryes } \\
\text { III }-\sigma_{0} & =10^{5} \text { baryes. }
\end{aligned}
$$

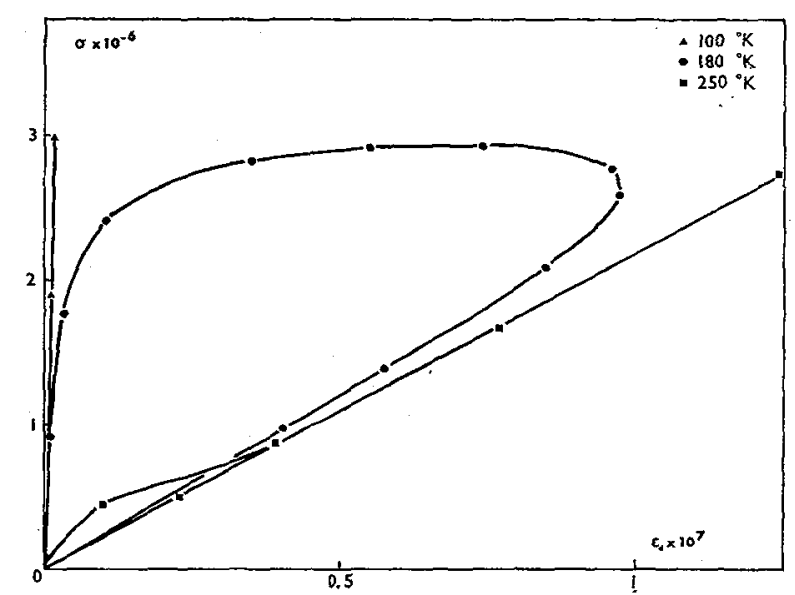

Fig. 5. - Cycles effort-déformation dus aux dislocations pour trois températures (désancrage type Friedel, $\sigma_{0}=3 \times 10^{6}$ baryes).

en fonction de la température. D'après les résultats de la figure 5 on peut calculer l'anomalie de module $\frac{\Delta E}{E}=\frac{\varepsilon d}{\varepsilon_{e}+\varepsilon_{d}}$. La figure 6 où l'on a tracé :

$$
\frac{\Delta E}{E}=f\left(\frac{\Delta T}{T}\right)
$$

met en évidence le fait que l'utilisation de la relation 1 est incorrecte dans le cas d'un tel phếnomène physique.

A titre de comparaison, nous avons donné les cycles correspondant au désancrage type TGL (Fig. 7, avec $N_{0}=3$ ). On remarque que pour

$$
\sigma<\sigma_{2}=0,6 \times 10^{6} \text { baryes, }
$$

toutes les dislocations sont à l'état complètement ancré (configuration 1) le cycle ne s'ouvre que pour $\sigma>\sigma_{2}$.

Il semble donc que les cycles effort-déformation obtenus dans le cas d'un désancrage type TGL ne correspondent pas aux résultats expérimentaux observés 


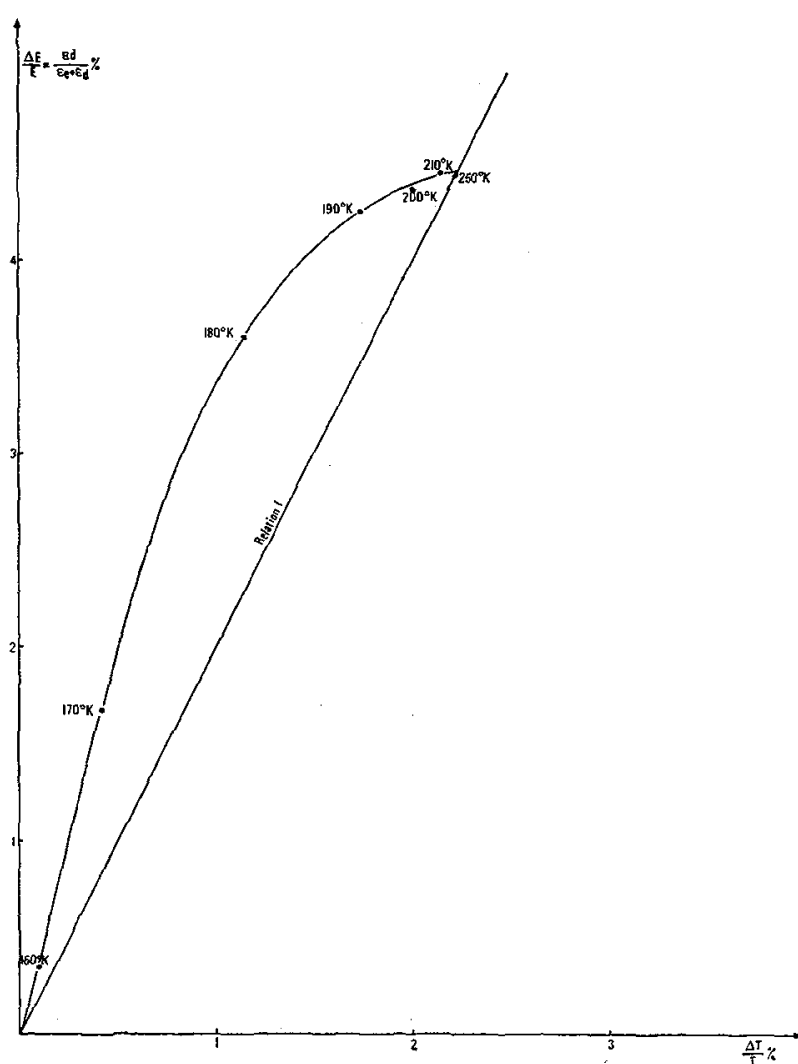

Frg. 6. - Anomalie de module en fonction de l'anomalie de période $\left(\sigma_{0}=3 \times 10^{6}\right.$ baryes $)$.

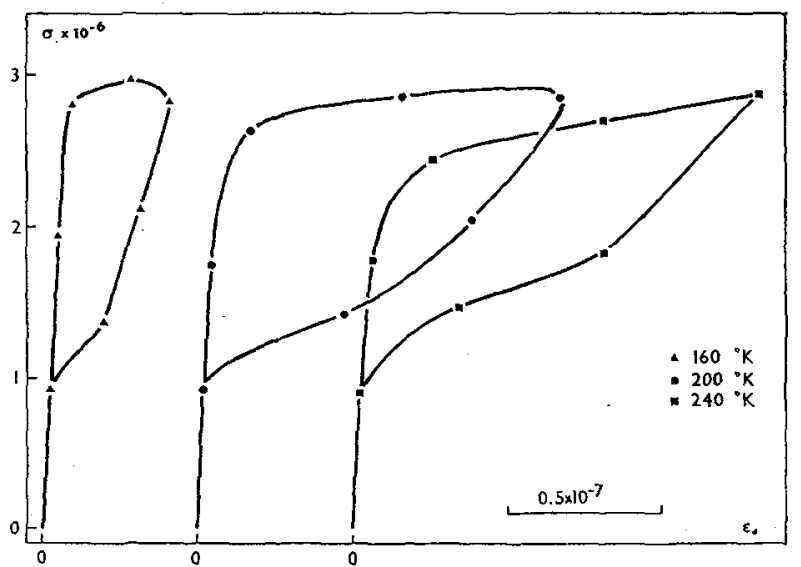

Frg. 7. - Cycle effort-déformation correspondant au désancrage type TGL $\left(N_{0}=3\right)$.

jusqu'ici (Roberts, Hartman, 1964). Avec les relations (7) de type Friedel les cycles obtenus semblent plus proches de la réalité.
D'autre part, la figure 8 montre les courbes $\delta$ et $\Delta T / T$ fonction de $\sigma_{0}$ pour trois températures. On voit que pour les températures inférieures ou supérieures à

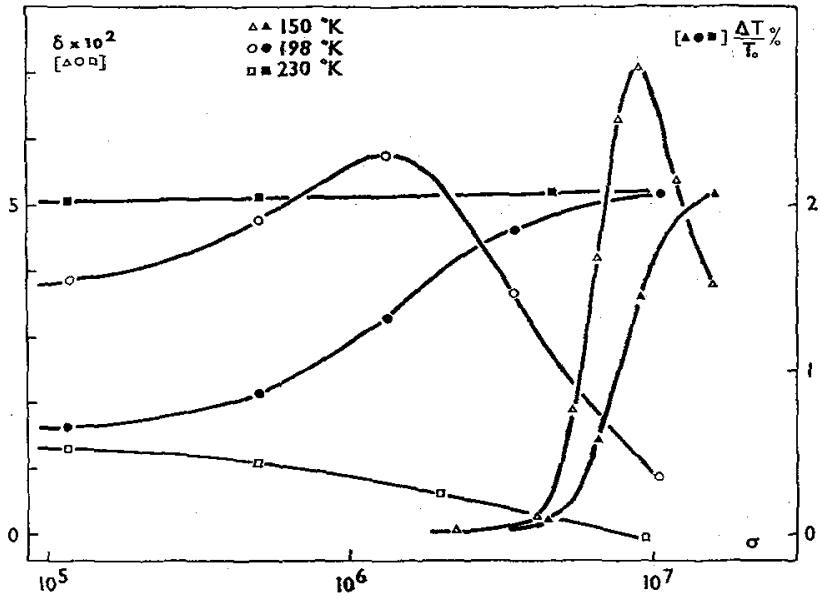

FIG. 8. - Courbes frottement intérieur et anomalie de période en fonction de $\sigma_{0}$ pour trois températures (désancrage type Friedel).

la température du pic correspondant à une valeur $\sigma_{0}$ donnée, le diagramme $\theta=f\left(\sigma_{0}\right)$ ne représente pas du tout le même aspect, contrairement à ce qui se passait pour le modèle de TGL (voir Peguin et Birnbaum, 1968) où pour $\sigma<\sigma_{2}$, le frottement intérieur était nul.

L'intérêt de cette étude est double :

- La méthode de calcul numérique permet un tracé simultané des courbes de frottement intérieur, d'anomalie de période et de cycles effort-déformation. Elle doit également permettre de les comparer aux résultats expérimentaux si les paramètres choisis sont réalistes. Par exemple : dans le cadre du modèle que nous avons étudié, il apparaît que le désancrage de type Friedel (1963) est plus proche de la réalité que celui de TGL (Tentonico, Granato, Lucke, 1964).

- Elle met en évidence la contradiction qui consiste à vouloir apprécier une anomalie de module par une mesure d'anomalie de période dans le cas d'un phénomène anélastique. La signification physique de l'anomalie de période est difficile à interpréter dans le cas général. Elle ne peut l'être que dans le cadre d'un modèle particulier. L'étude de l'évolution parallèle du frottement intérieur et de l'anomalie de période peut servir, plus correctement que le rapport $r$, à distinguer entre les pics de relaxation pure et d'autres comme ceux liés au désancrage thermomécanique des dislocations.

\section{Bibliographie}

[1] Delorme (J. F.), Perez (J.), Peguin (P.), Gobin (P.), (Cahier de Rhéologie, à paraître en 1970).

[2] Dienes (G. J.), Phys. Rev., 1952, 86, 228.

[3] Friedel (J.), Phil. Mag., 1953, 44, 444.
[4] FRIedel (J.), Conference Teddington Middlesex, 1963.

[5] Granato (A.), LuCke (K.), J. Appl. Phys., 1956, 27, 583.

[6] Granato (A.), Lucke (K.), Schlipf (J.), TeutoNICO (L.), J. Appl. Phys., 1964, 35, 2732. 
[7] Heuser (F.), Thesis 1965, Technishe Hochule, Aachen, Germany.

[8] Hikata (A.), Elbaum (C.), Phys. Rev., Letters., 1967, 18,750 .

[9] Hutchison (T. S.), Rogers (D. H.), J. Appl. Phys, 1962, 33, 792.

[10] Kolwa (M.), Hasiguti (R.), Act. Met., 1965, 13, 1219.

[11] Mason (W. P.), Phys. Rev., 1966, 143, 229.

[12] Melgailis (J.), Phys. Stat. Sol., 1966, 16, 247.

[13] Morrow (D.), « Internal friction, damping and cyclic plasticity 》, ASTM, 1965, 45.

[14] Motr (N. F.), Phil. Mag., 1952, 43, 1151.

[15] Peguin (P.), Perez (J.), Gobin (P.), Trans. AIME, 1967, 239, 438.

[16] Peguin (P.), Birnbaum (H. K.), J. Appl. Phys., 1968, 39.4428 .
[17] ReAD (W.), Phys. Rev., 1940, 58, 371.

[18] Robert (J. M.), Hartman (D. E.), Trans. AIME, 1964, 230, 1125.

[19] Rogers (D. H.), J. Appl. Phys., 1962, 33, 229.

[20] Stern (R.), Granato (A.), Act. Met., 10, 358.

[21] Teutonico (L.), Granato (A.), Lucke (K.), J. Appl. Phys., 1964, 35, 220.

[22] Timoshenko (S.), “ Théorie des vibrations », Lib. Ch. Béranger, Paris, 1954, 130.

[23] Tittman (B. R.), Bommel (H. E.), Phys. Rev., 1966, 151, 178.

[24] Vineyard (G.), J. Phys. Chem. Sol., 1957, 3, 121.

[25] ZeNER (C.), «Elasticité et anélasticité des solides 》, Dunod, 1955. 